\title{
Tasa de cambio y precios internos: Un modelo de organizacion Industrial
}

\author{
José Roberto Suay
}

\section{Introducción}

Tradicionalmente el análisis de las relaciones de precios en economías abiertas parte de la Ley de Un Solo Precio la cual afirma que existe un arbitraje perfecto entre los bienes comerciados que da lugar a la igualación de los precios en diferentes localidades excepto por desviaciones explicadas por la existencia de tarifas y costos de transporte.

De acuerdo a esta teoria, el precio de un bien en cualquier parte es el mismo cuando se expresa en una moneda común. De esa manera es válido escribir:

$$
p_{i}=e p_{i}^{*}
$$

donde $p_{i}$ es el precio en el mercado nacional del bien "i", e es la tasa de cambio entre la moneda nacional y extranjera, y $\dot{p}_{i}$ representa el precio en el extranjero del bien "i". Mientras que la anterior ecuación se conoce como la versión extrema de la teoría de la paridad del poder de compra (PPC), la versión moderada da origen a la idea de que cambios en la tasa de cambio se explican con relación a cambios en el nivel de precios relativos.

Normalmente esta teoría ha asumido que los precios relativos entre dos paises son independientes de la tasa de cambio, ya que la última solamente refleja pasivamente la tendencia inherente a los precios internos. Es asi como se asegura que movimientos en la tasa de cambio no pueden tener efectos permanentes en los precios relativos de un país. Sin embargo, estudios empíricos han mostrado que la tasa de cambio si afecta los precios relativos Por ejemplo, Frenkel (1978: 183) asegura que:

"se puede inferir de las pruebas de 'causalidad' entre tasas de cambio y razones de precios que la formulación tradicional de la PPC- que 
ve los precios como 'causa' de la tasa de cambio- no es estrictamente apoyada por los datos del periodo en consideración. Ellos implican que, si uno ha de utilizar el método de regresión, la especificación correcta comprende 'ecuaciones de precios' en las que razones de precios son las variables dependientes mientras que las tasas de cambio aparecen en el miembro derecho de la regresión"

Por otra parte, la PPC sufre adicionales limitaciones. La más seria de ellas es que únicamente postula la existencia de cierta relación entre tasas de cambio y precios internos sin especificar ningún mecanismo que explique cómo se da la relación y localice elementos importantes para el análisis empírico. Asi por ejemplo, de acuerdo a la teoría no hay manera de discernir sobre efectos diferenciales que cambios en la tasa de cambio puedan tener en los precios de productos fabricados en distintas industrias con diferente estructura económica (número de empresas, poder monopólico, nivel de componente importado en los insumos, etc).

El objetivo de este trabajo es presentar un modelo alternativo en el que movimientos en la tasa de cambio si tienen efecto en los precios relativos de un país. Asimismo, el modelo utilizado ofrece ciertas razones que explican por qué ciertas industrias se ven afectadas en mayor grado que otras por movimientos en la tasa de cambio. Finalmente, se trata derivar del análisis algunos elementos de política económica que ayuden a comprender mejor la dinámica que relaciona los sectores externo y doméstico de la economía.

\section{Un modelo de organización Industrial.}

El uso de modelos de organización industrial para el análisis de problemas de economía internacional es nuevo y se relaciona con la investigación de los efectos que la competencia imperfecta, economias de escala, barreras de entrada, diferenciación de producto, etc. tienen sobre el comercio internacional. Este nuevo enfoque pone de relieve algunas características novedosas para la teoría de la economía internacional:

- Aparecen nuevos aspectos de la competencia que se vuelven importantes para explicar los patrones del comercio internacional tales como los efectos que sobre ella tienen la calidad, la propaganda, los gastos de investigación y desarrollo, el comportamiento estratégico de las firmas, etc.

- Cuando se consideran mercados imperfectos, la existencia de renta monopolista crea un incentivo para el análisis y desarrollo de políticas económicas que permitan al gobierno de un país anexar dicha renta a sus propios residentes y aumentar el bienestar social. Esto da lugar a la elaboración de nuevas preguntas a resolver por 
el enfoque normativo.

- Debido a que diferentes gobiernos conducen su política comercial simultáneamente, aparece un área de análisis que se encarga de estudiar los resultados de la interacción estratégica entre gobiernos.

En general, las conclusiones del análisis se desvian de las establecidas por la teoría ortodoxa del comercio internacional. Así por ejemplo. Dixit (1984: 14) concluye:

"Hemos visto que las políticas apropiadas cuando el comercio ocurre en mercados oligopólicos difieren significativamente de las disefiadas para situaciones competitivas. La posibilidad de que un impuesto contrarrestante pueda ser deseable cuando un pais extranjero subsidie sus exportaciones, y de que cierto relajamiento o racionalización de la regulación anti-monopólica sea justificable son ejemplos sobresalientes"

En este sentido es importante explorar que nuevas perspectivas abren los modelos oligopólicos al análisis de la relación entre tasas de cambio y precios internos. El modelo que presentamos a continuación es una ampliación del elaborado por Dornbusch (1987), en donde se ha tratado de introducir insumos importados y de determinar efectos en la producción disponible en el mercado nacional.

\section{El modelo básico.}

Vamos a trabajar con un modelo sumamente sencillo sobre la estructura del mercado y el comportamiento de los productores. Por el lado de la demanda empezamos con un supuesto extremadamente simple: la demanda en el mercado es una función lineal del precio:

$$
Q_{d}=a-b p(1)
$$

Los productores, por su parte enfrentan costos unitarios constantes y utilizan solamente un insumo: las empresas nacionales pagan $w$ por dicho insumo y las extranjeras $\mathbf{w}^{*}$. Cada empresa compra insumos en su respectivo mercado nacional: las nacionales compran insumos domésticos y las extranjeras insumos extanjeros. Finalmente, asumimos que hay $n$ empresas nacionales y $n^{\star}$ empresas extranjeras compitiendo en el mercado nacional, y que las empresas nacionales son idénticas entre sí pero diferentes a las extranjeras. Esto implica que cada empresa nacional venderá la misma cantidad en el mercado que su competidora nacional, pero dicha cantidad será diferente a la de la competidora extranjera. Lo mismo se aplica para la empresa extranjera. De tal manera, 
podemos hablar de una firma representativa tanto nacional como extranjera y expresar la oferta en el mercado como:

$$
Q_{0}=n q+n^{*} q^{*}
$$

Asumidos también que el comportamiento de las firmas es consistente con el modelo de Cournot el cual asume que el empresario trata de maximizar su ganacia seleccionando la cantidad a producir bajo el supuesto de que la cantidad producida de sus competidores está dada y que permanecerá inalterada ante su decisión. Centrándonos - por el momento - en lo que sucede en el mercado nacional, podemos decribir el problema que confronta la firma representativa nacional y extranjera como maximar la función de ganancia con respecto a la cantidad que desean producir. Para la empresa nacional y extranjera, la función de ganancia es respectivamente:

$$
\begin{aligned}
& \pi_{i}=(p-w) q_{i} \\
& \pi_{i}^{*}=(p / e-w) q_{i}^{*}
\end{aligned}
$$

Recordando que en este caso el precio (p) es una función de la cantidad vendida en el mercado, podemos encontrar una expresión del precio de equilibrio en función de la cantidad usando las ecuaciones (1) y (2):

$$
p=\left(a-n q-n^{\star} q^{*}\right) / b(5)
$$

Debido a que en el modelo Cournot cualquier firma asume que la producción de sus competidores está dada y que no cambiará debido a las acciones propias, la ecuación (5) es vista por la firma representativa nacional y extranjera respectivamente como:

$$
\begin{aligned}
& p=\left[a-q_{i}-(n-1)-n^{*} q^{*}\right] \quad / b(5 a) \\
& p=\left[a-n q-q_{i}^{*}-\left(n^{*}-1\right) q^{*}\right] / b(5 b)
\end{aligned}
$$

en donde $\mathrm{q}$ y $\mathrm{q}^{*}$ son considerados como cosntantes.

Las condiciones de primer orden para maximización de la ganancia para la firma nacional y extranjera son:

$$
\begin{aligned}
& p=w+q_{i} / b \\
& p=e w^{*}+q_{i}^{*} / b
\end{aligned}
$$

Sumando a través de las $n$ firmas nacionales y $n^{\star}$ extranjeras se obtiene:

$$
n p+n^{\star} p=n w+n q / b+n^{\star} e w^{*}+n^{*} q^{\star} / b
$$

Arreglando términos y usando la condición de equilibrio de oferta $y$ demanda $\left(a-b p=n q+n^{\star} q^{\star}\right)$ :

$$
n p+n^{*} p+p=n w+n^{*} e w^{*}+a / b
$$


Finalmente obtenemos:

$$
\mathrm{p}=\left(n w+n^{*} e w^{*}\right) / N+a / b N
$$

en donde $\mathrm{N}=\mathrm{n}+\mathrm{n}^{*}+1$.

Hemos llegado a expresar el precio de mercado como función de las condiciones de producción y demanda. Nótese que el primer término del segundo miembro de la ecuación puede ser interpretado como el costo unitario ponderado de acuerdo a la participación de empresas nacionales y extranjeras en el mercado nacional. El segundo miembro, corrobora lo que el sentido común sugiere: el precio es directamente proporcional a la demanda autónoma e inversamente proporcional a la pendiente de la demanda.

Para analizar el efecto que cambios en la tasa de cambio tienen sobre el precio de mercado necesitamos calcular la elasticidad del precio interno con respecto a la tasa de cambio. Esta se define como:

$$
\phi=(d p / d e) \times(e / p)
$$

De la ecuación (7) obtenemos $\mathrm{dp} / \mathrm{de}$ y multiplicando por e/p tenemos:

$$
\phi=\left(n^{*} / N\right) \times\left(e w^{*} / p\right)
$$

El término $n^{*} / N$ representa la participación de empresas extranjeras en el mercado nacional; la expresión indica que una mayor participación de empresas extranjeras hará que los efectos de cambios en la tasa de cambio sean sentidos con mayor fuerza. Un $n^{\star} / \mathrm{N}$ grande puede tomarse como indicativo del poder monopólico que las empresas extranjeras tienen sobre nuestro mercado. Asi a mayor poder monopólico extranjero en el mercado nacional mayor será el efecto de una devaluación. Por otro lado, ew*/p es el inverso de la mark-up de las empresas extranjeras. Una mayor mark-up implica una menor elasticidad de nuestros precios a una depreciación o apreciación de nuestra moneda. Algo que es digno de mencionar, es que en cualquier caso la elasticidad de nuestros precios es positiva y menor de la unidad. Cambios en $\theta$ no se transmiten proporcionalmente a los precios, lo cual se debe a que los efectos que la tasa de cambio tiene sobre los precios internos viene ponderado por la participación de las empresas extranjeras en el mercado nacional; sin embargo, una devaluación slempre incrementará los precios.

Uno de los resultados del modelo - en su presente forma- que preocupa es que los efectos en los precios, prácticamente no dependen de las condiciones internas de producción: el único efecto que ellas tienen es a través de $N\left(n+n^{*}+1\right)$. La razón que explica esto es que cambios en e no afectan directamente las condiciones de rentabilidad de las empresas nacionales. Esto puede verse en las funciones de reacción de las firmas nacionales y extranjeras, como se presenta a continuación. 
La función de reacción de la firma nacional expresa la producción $q_{i}$ que maximiza sus ganancias $\pi_{i}$ para cualquier valor $q_{i}$ especificado. La función de reacción para la firma nacional y extranjera es respectivamente:

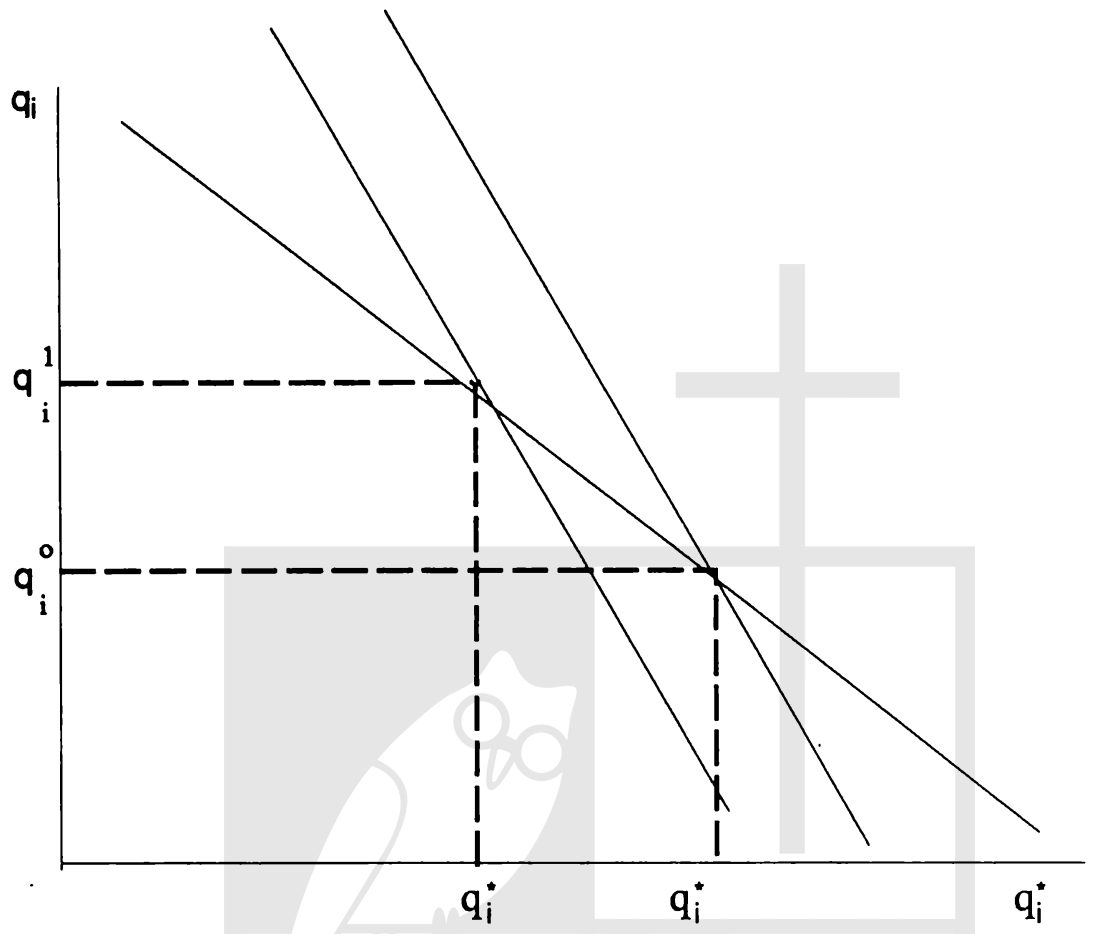

Gráfico No. 1

$$
\begin{aligned}
& q=\left(a-b w-n^{*} q^{*}\right) /(n+1) \\
& q=\left(a-b e w^{*}-\left(n^{*}+1\right) q^{*}\right] / n
\end{aligned}
$$

Las cuales en el caso particular son funciones lineales. Claramente puede apreciarse que la función de reacción de la empresa extranjera tiene una mayor pendiente que la de la firma nacional. Además de la ecuación (10a) se deduce que cambios en la tasa de cambio (e) no afectan la función de reacción de las firmas nacionales. El efecto se limita a las firmas extranjeras. Así por ejempo (Ver Gráfico 1), una depreciación de la moneda nacional (incremento en e), desplaza la función de reacción de la firma extranjera representativa hacia el origen, asi el resultado final es un incremento en las ventas de las firmas nacionales - de $q_{i}{ }_{i}$ a $q_{i}^{1}$. 
debido a la pérdida de mercado por parte de las extranjeras. La razón es que las firmas extranjeras ven reducidas sus ganancias a raíz de que las ventas en el mercado doméstico rinden menos en moneda extranjera.

De las funciones de reacción podemos obtener la expresión para el cambio en la cantidad vendida por las firmas nacionales y extranjeras debido a una depreciación:

$$
\begin{aligned}
& \mathrm{dq} / \mathrm{de}=\left(n^{*} / N\right) \times b w^{*} \\
& d q^{*} / d e=-[(n+1) / N] \times b w^{*}
\end{aligned}
$$

Asi el incremento de las ventas de las firmas nacionales debido a una devaluación será mayor cuánto mayor sea la participación en el mercado de las firmas extranjeras, la pendiente de la demanda y los costos unitarios en el extranjero. La elasticidad de la cantidad de las firmas nacionales con respecto a la tasa de cambio puede asimismo expresarse como:

$$
B=\left(n^{*} / N\right) \times\left(e w^{*} / p\right) \times(b \times p / q)
$$

La elasticidad tasa de cambio de la cantidad de las firmas domésticas será mayor entre mayor sea la participación y la mark-up de las firmas extranjeras en el mercado nacional, y la elasticidad precio de la demanda en el mercado nacional. La elasticidad es en general positiva y puede asumir valores fuera del intervalo $[0,1]$ de acuerdo a si la pendiente de la demanda es suficientemente grande. Esto significa que en cualquier caso, una devaluación beneficiaría a los productores nacionales en sus ventas.

Una pregunta que resulta interesante contestar es ¿Qué sucede con la cantidad vendida en el mercado? Para ello, supongamos que la oferta y demanda siempre están en equilibrio y que por tanto el cambio en la cantidad vendida puede calcularse mediante:

$$
d Q / d e=n d q / d e+n^{*} d q^{*} / d e
$$

Dado que $d q /$ de $y d^{*} / d e$ tienen signos opuestos, y como hay $n$ y $n^{*}$ firmas domésticas y extranjeras que por lo general $n \neq n^{*}$. es concecible que la cantidad en el mercado nacional permanezca constante si el incremento en la producción de las empresas nacionales compensa la reducción en la de las extranjeras. Sin embargo, en la generalidad de los casos esto no es posible. Veamos por qué.

Usando las ecuaciones (11a) y (11b) obtenemos:

$$
d Q / d e=-\left(n^{*} / N\right) \times b w^{*}
$$

Excluyendo el caso en que la elasticidad de demanda en el mercado nacional es cero $(b=0)$, de la expresión anterior se puede concluir que el resultado de una devaluación será siempre una disminución en la producción destinada al mercado interior. 
Esto presenta el dilema de ponderar la pérdida de bienestar en el consumidor en contra del aumento en las ganancias de los productores nacionales. Si se asume que esta industria comprende la totalidad de la economía nacional y que el estado está dispuesto a efectuar una política redistributiva que compense al consumidor por la pérdida de bienestar, podemos calcular el cambio en el bienestar de la sociedad observando que pasa con el excedente del consumidor y la ganancia del productor nacional. En tal caso, el bienestar aumentará si la pérdida en el excedente del consumidor es más que compensada por la ganancia extra de las empresas nacionales derivada del incremento en su participación en el mercado, lo cual obviamente dependerá en alguna manera de los costos nacionales. En este modelo, el bienestar aumentará si:

$$
d w / d e=\left(n^{*} w^{*} / N\right)\left[b(p-w) n-n^{*} q^{*}\right]>0
$$

\section{Insumos Importados}

Un caso importante en que la rentabilidad de las empresas nacionales se ve afectada por movimientos en la tasa de cambio es cuando la producción nacional necesita insumos importados. Hay dos formas en que se puede introducir esta complicación en nuestro modelo: una es a través de un segundo insumo el cual sería únicamente producido en el extranjero, la segunda consiste en asumir que la producción nacional del único insumo no alcanza a cubrir las necesidades de la industria nacional por lo que parte tiene que ser importada. A continuación desarrollaremos el segundo caso. En esta situación la función de ganancia del productor nacional puede escribirse como:

$$
\pi_{i}=\left[p-\infty w-(1-\infty) e w^{*}\right] q_{i}
$$

en donde $\infty$ representa la parte de la producción nacional que podria producirse si no pudiera comprarse el insumo en el mercado extranjero. En cierto sentido $\infty$ da una medida sobre la dependencia de la industria nacional con respecto al insumo extranjero. La condición de primer orden para la maximización es:

$$
p=\infty w+(1-\infty) e w^{*}+q ; b
$$

Dado que las condiciones de maximización para la empresa extranjera no cambian, y siguiendo el mismo proceso anterior podemos encontrar el precio de mercado (Ver apéndice para la derivación):

$$
p=\left\{\left[n \infty w+\left(n[1-\infty]+n^{*}\right) e w^{*} / N\right\}+a / b N\right.
$$

la elasticidad de los precios con respecto a la tasa de cambio:

$$
\phi=\left\{\left[n(1-\infty)+n^{\star}\right] / N\right) \times\left(e w^{*} / p\right)
$$


y la elasticidad de la cantidad vendida por las firmas nacionales con respecto a la tasa de cambio:

$$
B=\left\{\left[\left(n^{\star}+1\right) \infty-1\right] / N\right\} \cdot\left(e w^{\star} / p\right) \cdot(b p / q)
$$

La ecuación de los precios básicamente nos da la misma información que en el caso de insumos no importados. El precio es proporcional al costo unitario ponderado por la participación de las empresas nacional y extranjera en el mercado nacional, a la demanda autónoma e inversamente proporcional a la pendiente de la demanda. La elasticidad de los precios sigue siendo positiva pero ahora es mayor, ya que los cambios en la tasa de cambio no sólo afectan las ganancias de las empresas extranjeras sino que también los costos de las empresas nacionales. No obstante, el valor de $\phi$ no llega nunca a alcanzar el de la unidad.

Un resultado del modelo anterior que se ve modificado por la inclusión de materias primas importadas es que la elasticidad de la cantidad vendida por las empresas nacionales puede ser negativa: una devaluación puede afectar negativamente tanto las ventas de las firmas nacionales como extranjeras en el caso de que la cantidad de insumo importado por unidad de producto sea alta, en el presente modelo $B$ será negativa si $\infty$ $<1 / n^{*}+1$. El efecto sobre la ganancia de los productores nacionales es ambiguo, ya que dependerá de su habilidad de pasar el incremento en sus costos al consumidor.

\section{Implicaciones del Modelo.}

Se ha tratado de explorar como cambios en la tasa de cambio tienen repercusiones en los precios y las cantidades en una determinada industria. Hemos visto como los efectos dependen tanto de las condiciones de demanda como de la estructura de la industria (en este caso reflejada por el número de empresas y su distribución en empresas nacionales y extranjeras).

Una vez que dejamos de trabajar dentro del marco de una sola industria el modelo sugiere que industrias con distintas estructuras sufrirán de distinta manera los efectos de cambios en la tasa de cambio. Dichos efectos en última instancia traerán consigo redistribuciones del ingreso ya no sólo entre consumidores y productores sino también entre distintos grupos de productores.

De la misma manera, en lo que se refiere a materia de política cambiaria del modelo puede inferirse que el nivel de la tasa de cambio no sólo actua como discriminante entre importaciones y exportaciones sino también entre distintos tipos de importaciones y exportaciones. Asi ade- 
más puede concebirse que existe una tasa de cambio que será la óptima desde el punto de vista de cada industria, y que no tienen por qué coincidir con la que otras industrias consideren mejor. En otras palabras el modelo propugna por la existencia de tipo de cambio múltiple.

Un resultado importante del modelo es el hecho de que indica cómo los productores nacionales pueden verse perjudicados por una devaluación, y cómo - aún en el caso en que no existen materias primas importadas- no siempre serán capaces de ganar el mercado que las empresas extranjeras pierden. Por lo qué, aún cuando productores puedan ganar el consumidor se verá afectado por una menor disponibilidad de bienes y un encarecimiento de los mismos.

Es interesante también ver como este modelo no excluye la posibilidad de la existencia de estanflación. Por ejemplo, de acuerdo al modelo con insumos importados, el resultado de una devaluación es un incremento en el precio en el mercado nacional ( $\phi$ es positiva) a la vez que existe la posibilidad de una reducción en la cantidad producida por los productores domésticos ( $B$ es negativa si $\infty<1 / n^{*}+1$ ). Así cuando consideramos la existencia de industrias exportadoras a la par de las estrictamente orientadas hacia el mercado doméstico, es concebible que el efecto estimulante que una devaluación pueda tener en las empresas exportadoras no alcance a compensar por la caida de la producción nacional para el mercado doméstico. Así tendríamos que contracción en el producto puede acompanar a incremento en los precios.

Una aplicación del modelo es que puede permitir analizar los determinantes de los términos de intercambio. Siguiento a Dornbusch [1987], los términos de intercambio pueden definirse como:

$$
T=p / \mathrm{ep}^{*}
$$

en donde $p$ representa el precio a que los extranjeros compran nuestras exportaciones y ep* el precio que nosotros debemos pagar por nuestras importaciones.

Hallando d ( $p$ / ep*) / de podemos analizar los efectos de cambios en la tasa de cambio sobre los términos de intercambio:

$$
d\left(p / e p^{*}\right) / d e=\left[e p^{*} d p / d e-e p d p^{*} / d e-p p^{*}\right] /\left(e p^{*}\right)^{2}
$$

Multiplicando y dividiendo por e/p y por $e / p^{*}$ para expresar la derivada anterior en términos de elasticidades:

$$
d\left(p / e p^{*}\right) / d e=\left[p p^{*}\left(\phi-\phi^{*}-1\right)\right] /\left(e p^{*}\right)^{2}
$$

donde $\phi$ es la elasticidad de los precios domésticos ante un cambio en la tasa de cambio y $\phi^{*}$ es la análoga de los precios de nuestras importaciones. 
Claramente los términos de intercambio mejorarán si $\phi>\phi^{*}+1$ lo cual depende de la estructura oligopolística de los dos mercados en consideración. En el caso de una apreciación, los precios de nuestras exportaciones se incrementarán en relación con los de las importaciones si la competencia por importaciones en los mercados domésticos es restringida y si los mercados extranjeros son fuertemente dominados por nuestros productores.

Para concluir, unas palabras de advertencia. El modelo aquí desarrollando sufre de la tradicional crítica del modelo de Cournot: el comportamiento de los productores en que estos asumen que sus acciones no tendrán efecto en las de sus rivales es altamente implausible. Existen otros modelos que postulan diferentes hipótesis sobre el comportamiento de los productores, particularmente interesante es el de variaciones conjeturales. Sin embargo, con toda su sencillez el modelo anterior presenta posibles razones teóricas que explican el comportamiento de los precios de diferentes industrias ante fenómenos cambiarios. No obstante, el modelo anterior es únicamente adecuado para el análisis de corto plazo, esto particularmente se observa en que $n$ y $\mathrm{n}^{\star}$ son constantes en el modelo, lo que implica que la estructura del mercado esta dada y no se analizan los determinantes de ésta. Un análisis de largo plazo deberia incluir entre sus objetivos el estudio de los determinantes de la estructura económica.

\section{Apéndice I}

Las condiciones de primer orden y la función inversa de demanda para las $n$ y $n^{\star}$ empresas nacionales y extranjeras en el caso de que exista insumo importado son:

$$
\begin{array}{rlll}
n p & = & n / b \cdot q+ & n \propto w+n(1-\propto) e w^{*} \\
n p & = & n^{*} / b \cdot q+ & n^{*} e w^{*} \\
p & =a / b-n / b \cdot q-n^{*} / b \cdot q^{*} &
\end{array}
$$

Tomando el diferencial total:

$$
\begin{array}{rlrl}
n d p-n / b \cdot d q \quad & n \infty d W+n(1-\infty) e d W^{*}+n(1-\infty) w^{*} d e \\
n^{*} d p \quad n^{*} / b \cdot d q^{*} & = & e n^{*} d w^{*}+ & +n^{*} w^{*} d e \\
d p+n / b \cdot d q+n^{*} / b \cdot d q^{*} & =0 & 0
\end{array}
$$

Asumiendo que $d w=d w^{*}=0$

$$
\begin{array}{rlrl}
n d p-n / b \cdot d q & =n(1-\infty) w^{*} d e \\
n^{*} d p & n^{*} / b \cdot d q^{*} & = & n^{*} w^{*} d e \\
d p+n / b \cdot d q+n^{*} / b \cdot d q^{*} & = & 0
\end{array}
$$


En forma matricial y dividiendo por de:

$\left[\begin{array}{ccc}n- & -n b & 0 \\ n^{*} & 0 & -n^{*} / b \\ 1 & n / b & n^{*} / b\end{array}\right] \quad\left[\begin{array}{l}d p / d e \\ d q / d e \\ d q^{*} / d e\end{array}\right]=\left[\begin{array}{c}n(1-\infty) w^{*} \\ n^{*} w^{*} \\ 0\end{array}\right]$

El determinante de la matrix de coeficientes de las derivadas con respecto a la tasa de cambio es:

$r=n n^{*} / b^{2} \cdot\left(n+n^{*}+1\right)$

Usando la regla de Cramër podemos hallar:

$$
\begin{aligned}
& \mathrm{dp} / \mathrm{de}=\left[\mathrm{n}(\mathrm{n}-\propto) \mathrm{n}^{*} / \mathrm{N}\right] \cdot \mathrm{w}^{*} \\
& \mathrm{dq} / \mathrm{de}=\left\{\left[\left(\mathrm{n}^{*}+1\right) \propto-1\right] / \mathrm{N}\right\} \cdot \mathrm{bw}^{*} \\
& \mathrm{dq} / \mathrm{de}=-[(1+\infty n) / N] \cdot b w^{*}
\end{aligned}
$$

\section{Bibllografía}

Dixit, Avinash. International trade pollcy for ollgopollstic Industrles. The Economic Journal. Suppl. 1984, Vol. 94, pp. 1-16. Compartive Statics for oligopoly. International Economic Review. Vol. 27, no. 1, Febrero 1986.

Dixit, $A$. and Stem. Oligopoly and welfare: unlfled presentation with apllcatlons to trade and development. Europen Economic Review, vol 19, 1981, pp. 123-43.

Dixit, 'A. K. and Stiglitz, J. E. Monopollstic Competition and Optlmun Product Diverslty. The American Economic Review. Junio 1977, Vol. 67, no. 3. pp. 297-308.

Dornbusch, Rudiger. Exchange rates and prices. American Economic Review. Vol 77. no. 1, Marzo 1987. pp. 93-106.

Frenkel, Jacob A. Purchasing power parity: doctrinal perspectlve and ovidence from the 1920s. Journal of Intemational Economics 8 (1978): $169-191$.

Greenaway, David and Chris Milner. The Economics of Intra-Industry Trade. Basil Blackwell.

Genberg, Hans. Purchasing power parity under fixed and flexible exchange rates. Journal of International Economics, 8 (1978): 247-276.

Kravis, Irving and Lipsey, Robert. Price behavior in the light of balance of payments theorles. Journal of Intemational Economics 8 (1978): 193 -246.

Krugman, P. R. Increasing returns, monopolistic competition and International trade. Journal of International economics, vol 9, 1979, pp 469-79. Scale economles, product differentlation and the msttern of trade. American Economic Review, vol 70. 1980, pp. 950-59.

Lancaster, Kelvin Intraindustry trade under perfect monopolistic competition. Journal of International Economics, vol 10. 1980, pp. 151-175.

Salop S. C. Monopollstic competition with outside goods. The Bell Journal of Economics. Spring 79, Vol. 10, No. 1 pp. 141-156. 